\title{
Interleukin-17-dependent CXCL13 mediates mucosal vaccine-induced immunity against tuberculosis
}

\author{
R Gopal $^{1}$, J Rangel-Moreno ${ }^{2}$, S Slight ${ }^{1}$, Y Lin $^{1}$, HF Nawar $^{3}$, BA Fallert Junecko ${ }^{4}$, TA Reinhart ${ }^{4}$, J Kolls ${ }^{5}$, \\ TD Randall ${ }^{2,6}$, TD Connell ${ }^{3}$ and SA Khader ${ }^{1}$
}

The variable efficacy of tuberculosis (TB) vaccines and the emergence of drug-resistant strains of Mycobacterium tuberculosis (Mtb) emphasize the urgency for not only generating new and more effective vaccines against TB but also understanding the underlying mechanisms that mediate vaccine-induced protection. We demonstrate that mucosal adjuvants, such as type II heat labile enterotoxin (LT-Ilb), delivered through the mucosal route induce pulmonary Mtbspecific T helper type 17 (Th17) responses and provide vaccine-induced protection against $M$ tb infection. Importantly, protection is interferon- $\gamma$ (IFN $\gamma$ )-independent but interleukin-17 (IL-17)-dependent. Our data show that IL-17 mediates $\mathrm{C}-\mathrm{X}-\mathrm{C}$ motif chemokine ligand 13 (CXCL13) induction in the lung for strategic localization of proinflammatory cytokineproducing $\mathrm{CXCR}^{+}$(C-X-C motif chemokine receptor 5-positive) Tcells within lymphoid structures, thereby promoting early and efficient macrophage activation and the control of $M t b$. Our studies highlight the potential value of targeting the IL-17-CXCL13 pathway rather than the IFN $\gamma$ pathway as a new strategy to improve mucosal vaccines against TB.

\section{INTRODUCTION}

Mycobacterium tuberculosis (Mtb), the causative agent of tuberculosis (TB), infects one-third of the world's population and kills $>1.7$ million people worldwide every year. Although most infected individuals develop latent TB, the disease will reactivate in $5-10 \%$ of latently infected individuals. Therefore, efforts to design improved vaccines against $\mathrm{TB}$ are a research priority. Primary immunity to $M t b$ infection in humans and mice is thought to be dependent on T helper type 1 (Th1) cells that produce interferon gamma (IFN $\gamma$ ) and tumor necrosis factor alpha (TNF $\alpha)$; both cytokines that activate macrophages within the granulomas to control intracellular $M t b$ growth. ${ }^{1}$ However, current vaccination approaches that target Th1 responses have not been successful in enhancing protection against $M t b,{ }^{2-4}$ suggesting that additional pathways may need to be targeted to improve vaccine-induced immunity to TB.
The TB granuloma is formed in response to $M t b$ infection in the lung and is an essential component of immune protection. Interestingly, active TB granulomas contain organized lymphoid structures known as inducible bronchus-associated lymphoid tissue (iBALT), and induction of BBALT is mediated, in part, by the expression of C-X-C motif chemokine ligand 13 (CXCL13), ${ }^{5-8}$ which controls the formation of B-cell follicles, T-cell placement, and the optimal activation of macrophages for $M t b$ control. $^{6,8}$ Furthermore, pulmonary CXCL13 expression and iBALT formation are controlled by interleukin (IL)-17. ${ }^{7}$ Therefore, despite a dispensable role for IL-23 and IL-17 in primary immunity to TB, ${ }^{9} \mathrm{IL}-23$-dependent Th17 cells have a key role in Th1 recruitment and vaccine-induced protection in a parenteral model of vaccine-induced immunity to $M t b$ infection. ${ }^{10}$ Some mucosal vaccines are thought to provide superior protection against $M t b$ when compared with parenteral vaccination; ${ }^{11-13}$ however, the absolute requirement

\footnotetext{
${ }^{1}$ Department of Pediatrics, Division of Infectious Diseases, University of Pittsburgh School of Medicine, Pittsburgh, Pennsylvania, USA. ${ }^{2}$ Department of Medicine, Division of Allergy, Immunology and Rheumatology, University of Rochester Medical Center, Rochester, New York, USA. ${ }^{3}$ Witebsky Center for Microbial Pathogenesis and Immunology and Department of Microbiology and Immunology, University at Buffalo, Buffalo, New York, USA. ${ }^{4}$ Department of Infectious Diseases and Microbiology, University of Pittsburgh, Pittsburgh, Pennsylvania, USA. ${ }^{5}$ Richard King Mellon Institute for Pediatric Research, Department of Pediatrics and Immunology, University of Pittsburgh School of Medicine, Pittsburgh, Pennsylvania, USA and ${ }^{6}$ Department of Medicine, Division of Clinical Immunology and Rheumatology, University of Alabama at Birmingham, Birmingham, Alabama, USA. Correspondence: SA Khader (Shabaana.Khader@chp.edu)
} 
of the Th1 and Th17 pathways and the mechanism that mediates mucosal vaccine-induced protection against $M t b$ challenge is not known. In the current study, we demonstrate that the mucosal adjuvant, type II heat labile enterotoxin (LT-IIb), ${ }^{14}$ when delivered mucosally with an $M t b$-specific antigen, induces pulmonary Th17 and Th1 responses and provides vaccine-induced protection against $M t b$. Importantly, we show that vaccine-induced immunity against $M t b$ infection is IFN $\gamma$-independent and, in Ifng ${ }^{-/-}$mice, is dependent on IL-17. We also show that Th17 responses in vaccinated mice are associated with IL-17-dependent induction of CXCL13 within the lung parenchyma, promoting lymphoid structure formation and organization, strategic positioning of $\mathrm{T}$ cells within the lung, and maximal macrophage activation. These findings demonstrate that the immune mechanisms involved in successful primary and recall responses to $\mathrm{TB}$ are distinct and provide new evidence that targeting the IL-17-CXCL13 pathway by mucosal vaccination has the potential to improve vaccine strategies against $\mathrm{TB}$.

\section{RESULTS}

IL-17, but not IFN $\gamma$, is critical for mucosal vaccine-induced immunity against $M$ tb

Given that some mucosal vaccines are thought to mediate superior protection against $M t b$ when compared with parenteral vaccination routes, ${ }^{11-13}$ it is critical to determine whether Th1 or Th17 responses or both are required to mediate mucosal vaccine-induced protection against TB. Wild-type C57BL/6J (B6) mice intranasally vaccinated with an $M t b$ immunodominant $\mathrm{I}-\mathrm{A}^{\mathrm{b}}$-restricted peptide, early secreted antigenic target $\left(\right.$ ESAT6 $_{1-20}$ ) in combination with LT-IIb, a potent and well-characterized mucosal adjuvant, ${ }^{14}$ exhibited protection when challenged with $M t b$ when compared with sham-vaccinated $M t b$-challenged mice (Figure 1a). Importantly, protection was maintained following $M t b$ challenge in mucosally vaccinated mice that were rested long term ( $>100$ days) (Figure $\mathbf{1 b}$ ). We found that protection in mucosally vaccinated $M t b$-challenged lungs was associated with inflammatory lesions associated with well-organized lymphoid structures harboring distinct B-cell follicles interspersed with $\mathrm{CD}^{+} \mathrm{T}$ cells (Figure 1c). Furthermore, protection detected in mucosally vaccinated $M t b$-challenged mice was dependent on generation of $M t b$ specific $\mathrm{CD}^{+}{ }^{+} \mathrm{T}$ cells, as mice mucosally vaccinated with ESAT6 $6_{1-20}$ peptide with LT-IIb but not sham-vaccinated mice were protected when challenged with $M t b$ (Figure 1a). Consistent with this, we found that mucosal boost-regimes with ESAT6 $6_{1-20}$ in LT-IIb primed robust lung-resident ESAT6 $6_{1-}$ ${ }_{20}$-specific Th17-cell responses, while inducing a small but detectable lung-resident ESAT6 $6_{1-20}$-specific Th1 response (Figure 1d). In addition, enhanced lung-resident memory Th17 responses were maintained until day 100 postimmunization in lungs of mucosally vaccinated mice (Figure 1e). Upon challenge with $M t b$, we found that a significant population of activated $\mathrm{CD}^{+} \mathrm{T}$ cells that accumulated early in the lungs of mucosally vaccinated mice produced IL-17 (Supplementary Figure S1a), TNF $\alpha$ (Supplementary Figure S1b), and coproduced IFN $\gamma$ and TNF- $\alpha$ (Supplementary Figure S1c); interestingly, the activated $\mathrm{CD}^{+}{ }^{+} \mathrm{IFN} \gamma^{+}$-cell-producing population did not increase significantly in the lung (Supplementary Figure S1d). In addition, we found that activated $\mathrm{CD}^{+}{ }^{+} \mathrm{T}$ cells that accumulated early in the lungs of vaccinated $M t b$-challenged mice also expressed the chemokine receptors $\mathrm{C}-\mathrm{X}$-C motif chemokine receptor 3 (CXCR3) and CXCR5 (Supplementary Figure S1e,f). On day 15 post-Mtb challenge, we found higher ESAT6 ${ }_{1-20}$-specific Th17-cell responses when compared with Th1-cell responses (Figure 1f), and this coincided with higher induction of IL-17 mRNA when compared with IFN $\gamma$ mRNA in cells isolated from mucosally vaccinated $M t b$-challenged lungs (Figure 1g). Together, these data suggest that mucosal vaccination with ESAT6 $6_{1-20}$ and LT-IIb induces robust Th17 recall responses, enhances lymphocytic infiltration into lung parenchyma, and mediates vaccine-induced protection against $M t b$ challenge.

To determine whether IL-17 or IFN $\gamma$ production by vaccineinduced $\mathrm{CD}^{+}{ }^{+} \mathrm{T}$ cells was important for mucosal vaccineinduced protection, we next mucosally vaccinated $\mathrm{Ifng}^{-/-}$and $I l 17^{-1-}$ mice and challenged them with $M t b$. We found that, even though Ifng ${ }^{-/-}$mice had higher $M t b$ burden in the lungs, mucosal vaccination provided protection upon challenge at day 30 post-challenge (Figure 2a). In addition, vaccine-induced protection was maintained in Ifng ${ }^{-/-}$-vaccinated mice until day 45 (Figure 2b), at which point the unvaccinated Ifng ${ }^{-/-}$ mice died. Vaccine-induced protection was maintained until day 60 in B6-vaccinated mice (Figure 2c), contrasting with the lack of protection at both early and time points in $I l 17^{-/-}$ mucosally vaccinated mice (Figure 2a,c). Furthermore, vaccinated Ifng ${ }^{-/-}$mice exhibited robust Th17 recall responses (Figure 2d). By contrast, even though $I l 17^{-/-}$ mice generated a normal Th1 recall response (Figure 2e), they did not generate vaccine-induced immunity against $M t b$ infection (Figure 2a,c). A role for IL-17 in vaccine-induced immunity to $M t b$ was also confirmed by neutralizing IL-17 in mucosally vaccinated B6 mice (Figure $2 \mathrm{f}$ ). In addition, mucosal vaccination of mice that lack signal transducer and activator of transcription factor 3 (STAT3) in CD4 ${ }^{+}$T cells (Stat3.Cd4 $4^{-1-}$ mice) resulted in induction of antigen-specific Th1 responses (IFN $\gamma$ and $\mathrm{TNF} \alpha$ ) but not lung-resident Th17 responses (Figure 2g). Coincident with lack of induction of Th17 responses in Stat3.Cd4 ${ }^{-/-}$mice, mucosal vaccination did not confer vaccine-induced immunity upon $M t b$ challenge (Figure 2h). Finally, we show that neutralization of IL-17 in Ifng ${ }^{-/-}$-vaccinated mice also resulted in loss of vaccineinduced protection (Figure 2i). Therefore, our data indicate that, despite a requirement for IFN $\gamma$ in protection against primary $M t b$ infection, ${ }^{15,16}$ targeting IFN $\gamma$ in mucosal vaccine strategies may not be beneficial for improving protection against $M t b$ infection. Instead, our data show that IL-17 is a critical mediator of vaccine-induced immune protection following $M t b$ infection and that targeting the IL-17 pathway is likely to improve existing vaccine strategies against $M t b$. 
a
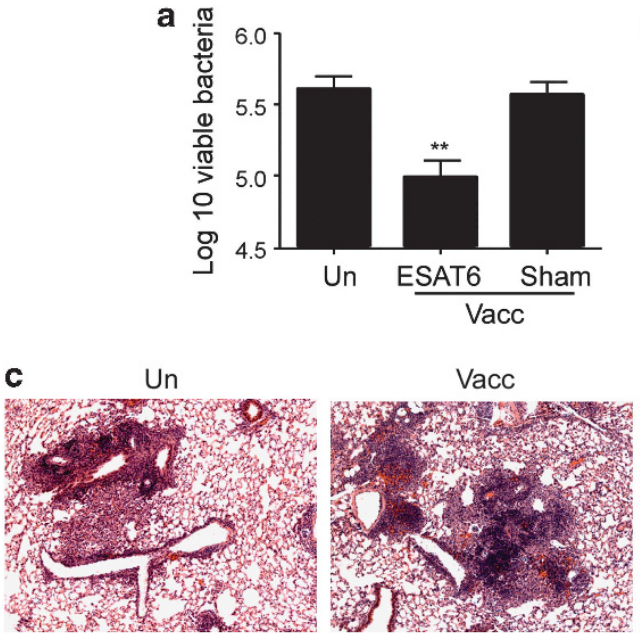

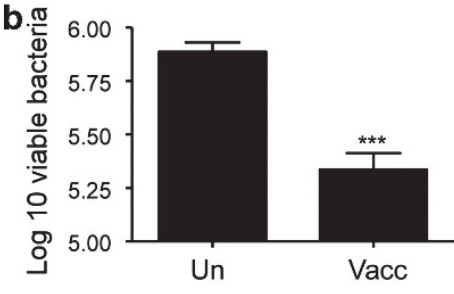

Un
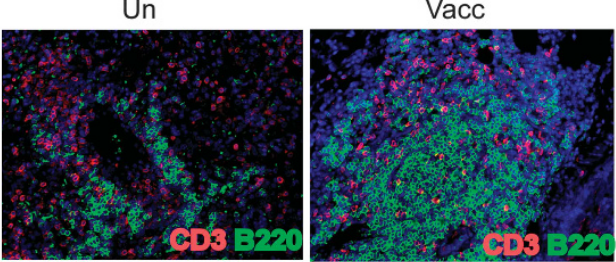

e

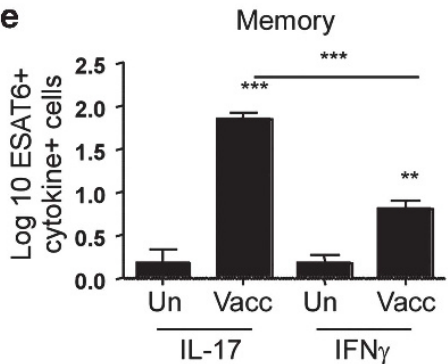

g

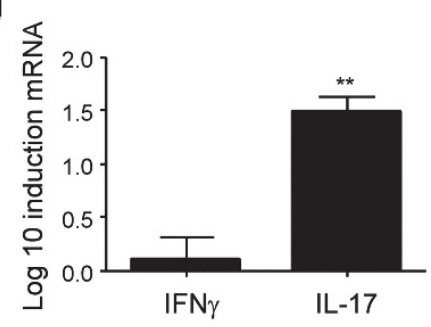

Figure 1 Mucosal vaccination induces vaccine-induced protection and robust T helper type 17 responses following Mycobacterium tuberculosis (Mtb) challenge. B6 mice were vaccinated mucosally vaccinated and boosted with ESAT6 ${ }_{1-20}$ in combination with LT-IIb (type II heat labile enterotoxin; Vacc-ESAT6) or sham vaccinated (Vacc-Sham) via the intranasal route. Control unvaccinated mice were also included (Un). Mucosally vaccinated B6 mice were rested for (a) 30 days or (b) 100 days, infected with aerosolized Mtb (100 colony-forming units), and lung bacterial burden was determined on day 30 post-infection. On day 30 post-infection, lungs were fixed in 10\% formalin, embedded in paraffin, and inflammatory lesions and lymphoid structure formation were assessed in formalin-fixed lungs by staining with (c-left panel) hematoxylin and eosin $(\mathrm{H} \& \mathrm{E})$; or (c-right panel) CD3 (red) and B220 (green). Original magnification for H\&E sections, $\times 100$; and immunofluorescence staining, $\times 200$.

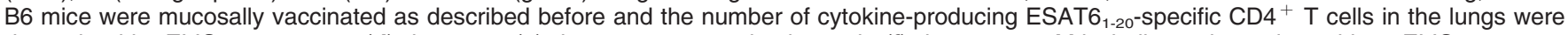
determined by ELISpot assay on (d) day 14 or (e) day 100 post-vaccination or in (f) day 15 post-Mtb-challenge by antigen-driven ELISpot assay. The Log10-fold induction of (interferon- $\gamma$ ) IFN $\gamma$ and interleukin (IL)-17 mRNA was determined in cells isolated from mucosally vaccinated $M$ Mtb-challenged lungs when compared with levels expressed in cell isolated from unvaccinated $M t b$-challenged lungs by (g) reverse transcriptase-PCR. The data points represent the mean ( \pm s.d.) of values from $4-6$ mice $(\mathbf{a}-\mathbf{g}) .{ }^{* *} P \leqslant 0.005,{ }^{* *} P \leqslant 0.0005$. One experiment representative of two is shown.

\section{IL-17 drives lung CXCL13 expression and mediates vaccine-induced protection against TB}

As protection in mucosally vaccinated mice was associated with ectopic lymphoid structure formation (Figure 1), we next determined whether IL-17-dependent vaccine-induced protection following $M t b$ challenge was associated with the development of organized lymphoid structures populated with lymphocytes. We found that mucosally vaccinated $I l 17^{-/-}$ mice formed small inflammatory lesions with disorganized lymphocytic infiltrates (Figure 3a,c upper panel) and containing small B-cell follicles (Figure 3b,c lower panel). By contrast, B6 and Ifng ${ }^{-/-}$-vaccinated mice developed distinct inflammatory lesions with rich lymphocytic infiltrates (Figure 3a,c upper panel), featuring wellorganized extensive B-cell follicles (Figure 3a,c lower panel), both of which were lost upon neutralization of IL-17 (Figure 3d-f). Furthermore, in support of the role of IL-17 produced by CD $4{ }^{+}$Th17 cells in induction of organized 


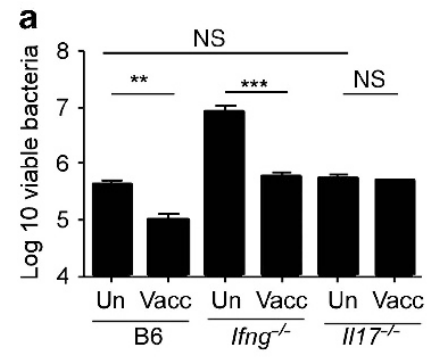

b
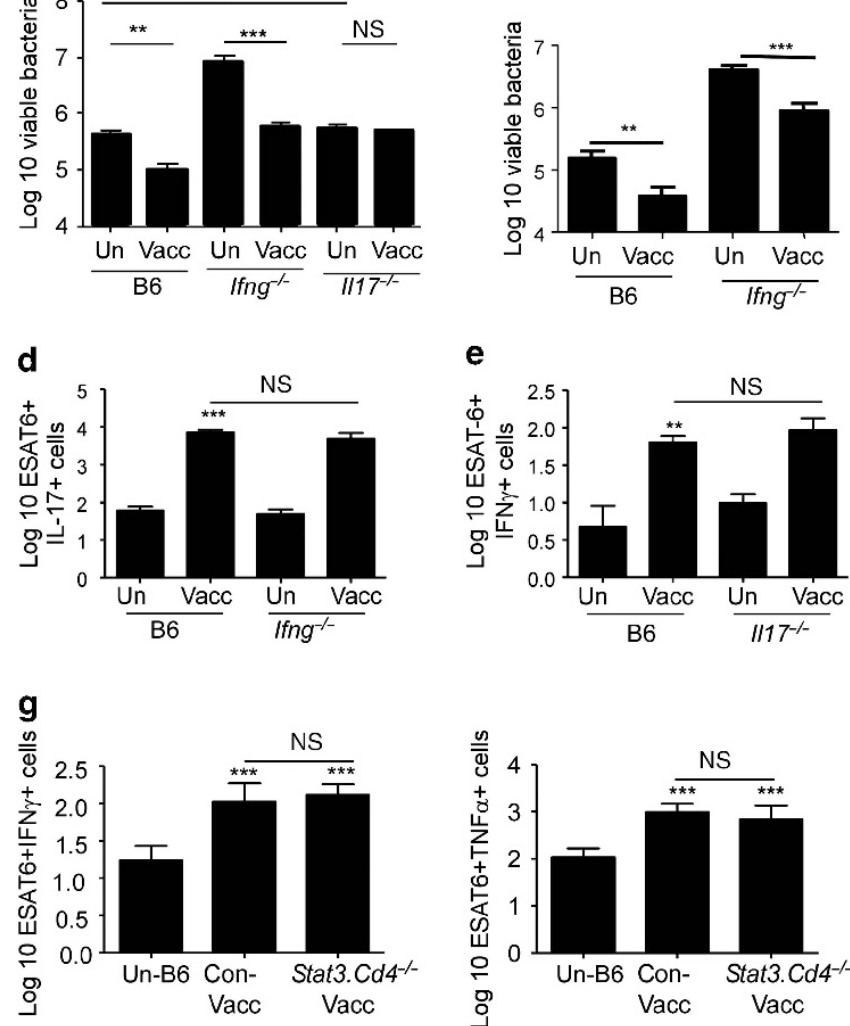

e
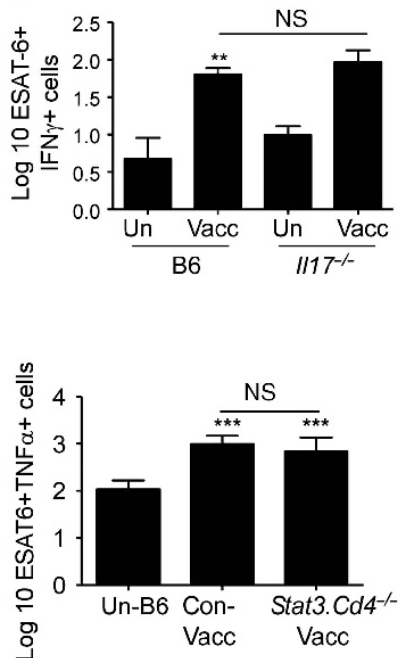

C
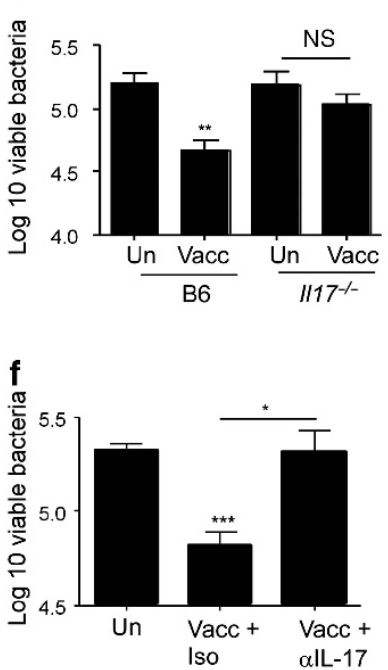
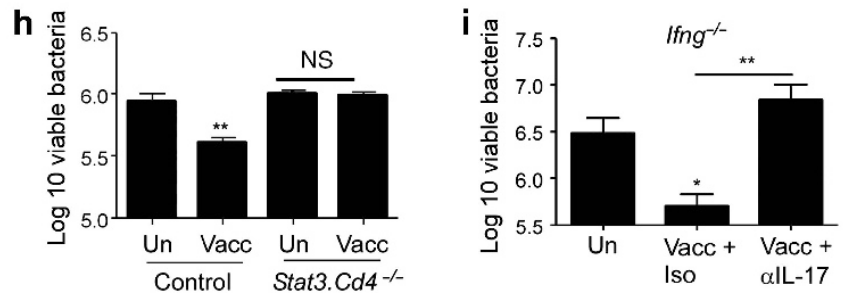

Figure 2 Interleukin (IL)-17, but not interferon- $\gamma$ (IFN $\gamma$ ), is crucial for vaccine-induced mucosal immunity against Mycobacterium tuberculosis $(\mathrm{Mtb})$ infection. B6, Ifng ${ }^{-1-}$ and $/ 117^{-/-}$were left unvaccinated (Un) or mucosally vaccinated (Vacc) with early secreted antigenic target (ESAT6 ${ }_{1-20}$ ) in combination with LT-Ilb (type II heat labile enterotoxin) and rested for 30 days. Subsequently, mice were challenged with $\sim 100$ colony-forming units Mtb by the aerosol route, and the lung bacterial burden was determined on (a) day 30 post-infection, (b) day 45 or (c) day 60 . The number of cytokine-producing ESAT6 ${ }_{1-20}$-specific CD4 ${ }^{+} \mathrm{T}$ cells in the lungs were determined by ELISpot in (d) B6 and Ifng ${ }^{-/-}$, (e) B6 and $/ / 17^{-/-}$mucosally vaccinated $M$ tb-challenged mice on day 15 post-infection. B6 mice were mucosally vaccinated, rested for 30 days and challenged with $M$ tb, following which they were treated with isotype control antibody or IL-17-neutralizing antibody between day 5 and day 21 ( $100 \mu \mathrm{g}$ per mouse every $48 \mathrm{~h}$ ), and (f) lung bacterial burden was determined on day 30 post-infection. (g) Stat3.Cd4 ${ }^{-1}$ mice or control littermates (Control) were vaccinated with ESAT6 ${ }_{1-20}$ in LT-Illb or left unvaccinated and the number of cytokine-producing ESAT6 ${ }_{1-20}$-specific CD4 ${ }^{+}$T cells in the lungs were determined by ELISpot on day 15 post-mucosal vaccination. Mucosally vaccinated Stat3.Cd4 ${ }^{-1-}$ mice or control littermate (Control) mice were rested for 30 days and challenged with $\sim 100$ colony-forming units Mtb by the aerosol route, and (h) the lung bacterial burden was determined on day 30 post-infection. Ifng $^{-1-}$ mice were vaccinated, rested for 30 days, challenged with Mtb following which they were treated with isotype control antibody or IL-17 neutralizing antibody between day 5 and day 21 ( $100 \mu$ per mouse every $48 \mathrm{~h}$ ), and (i) the lung bacterial burden was determined on day 30 post-infection. The data points represent the mean ( \pm s.d.) of values from 4-6 mice $(\mathbf{a}-\mathbf{i})$. ${ }^{*} P \leqslant 0.05 .{ }^{* *} P \leqslant 0.005 .{ }^{* * *} P \leqslant 0.0005$, NS, not significant. One of two independent experiments shown. STAT, signal transducer and activator of transcription factor; TNF, tumor-necrosis factor.

iBALT structures, both lymphocytic infiltrates (Supplementary Figure S2a,c upper panel) and B-cell follicles (Supplementary Figure S2b,c lower panel) were poorly organized in mucosally vaccinated $M t b$-challenged Stat3.Cd4 $4^{-1}$ mice. These data strongly suggest that IL-17 production by Th17 cells is required to efficiently form and organize lymphocytic infiltrates within inflammatory lesions for control of $M t b$ in mucosally vaccinated mice.
We recently showed that IL-17 was required for formation of iBALT structures in neonate lungs via the induction of CCL19 and CXCL13 following lipopolysaccharide-induced inflammation. ${ }^{7}$ In addition, CXCL13 is induced in the lung following $M t b$ infection, and the absence of CXCL13 results in impaired T-cell localization within the inflammatory lesions, decreased activation of lung macrophages, and increased susceptibility to Mtb infection. ${ }^{6}$ Therefore, we hypothesized that the critical role for 
a

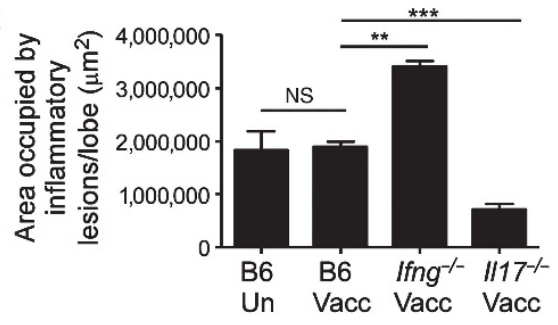

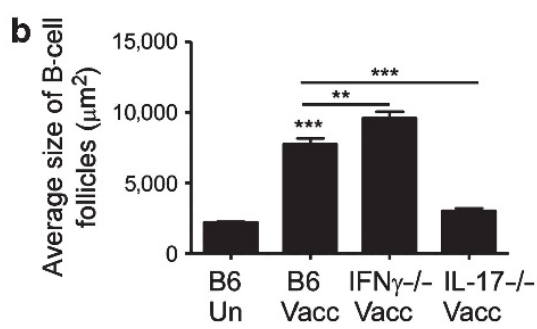

c
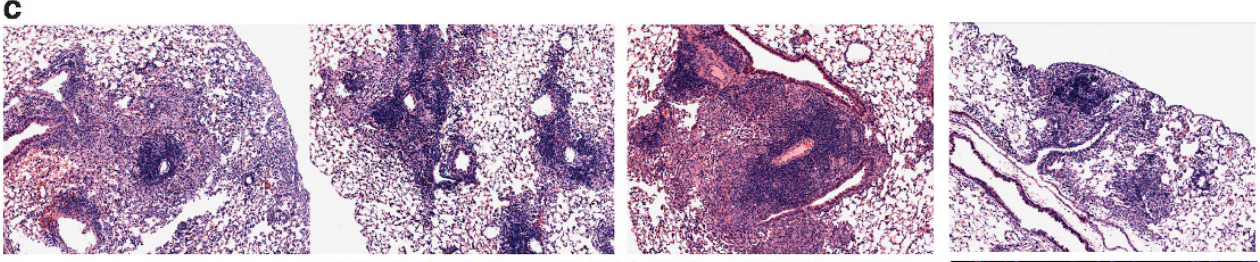

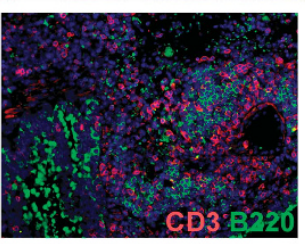

B6-Un

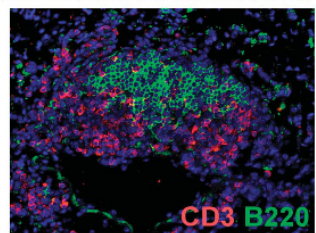

B6-Vacc

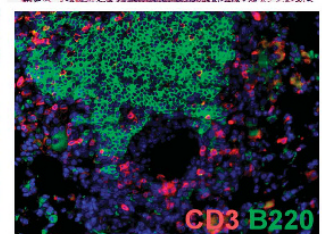

Ifng ${ }^{-/}$Vacc

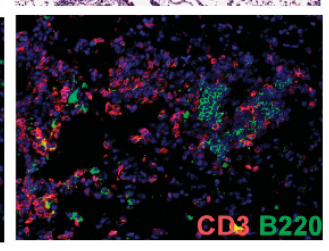

$1 / 17^{-/-}$Vacc

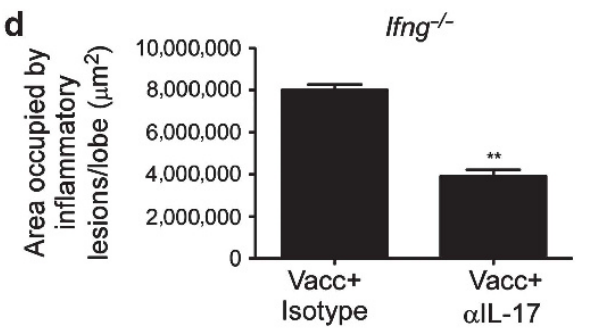

f
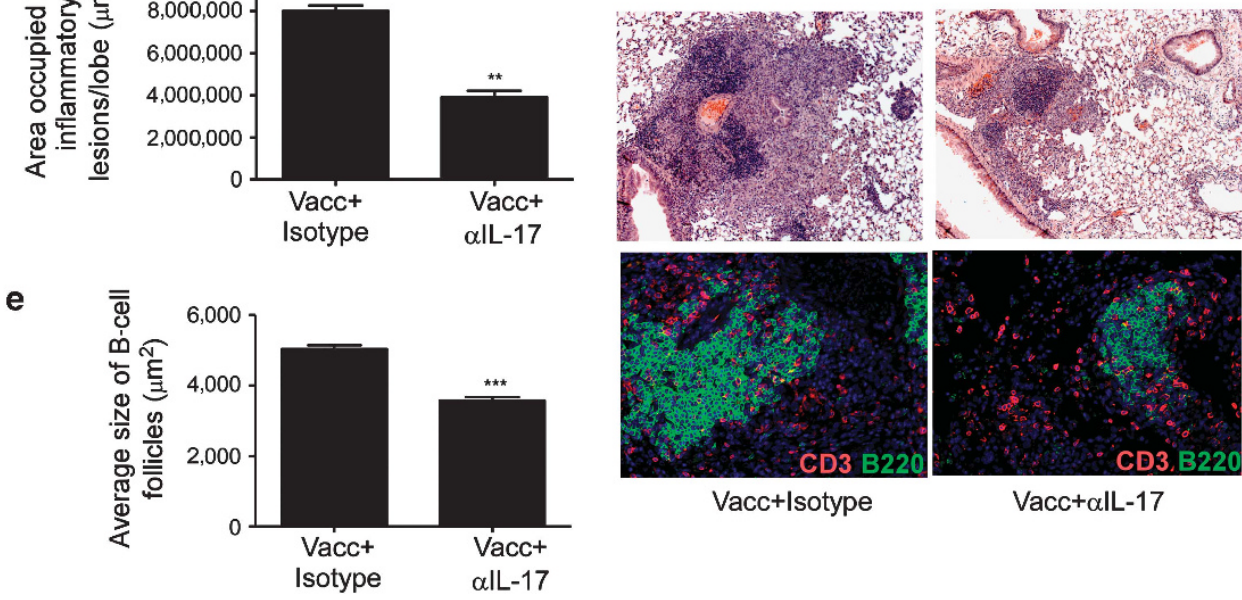

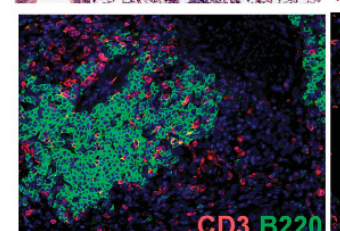

Vacc+Isotype

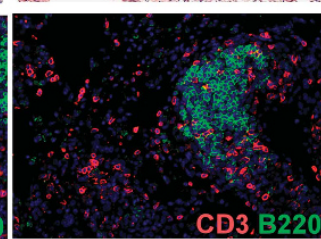

Vacc $+\alpha \mathrm{lL}-17$

Figure 3 Interleukin (IL)-17, but not interferon- $\gamma$ (IFN $\gamma$ ), is crucial for lymphocytic infiltration, B-cell lymphoid follicle, and granuloma formation in lungs of mucosally vaccinated Mycobacterium tuberculosis $(M t b)$-challenged mice. B6, Ifng ${ }^{-1-}$, and $/ 117^{-/-}$were mucosally vaccinated with ESAT6 ${ }_{1-20}$ (early secreted antigenic target) in combination with LT-Ilb (type II heat labile enterotoxin; Vacc) and rested for 30 days, then challenged with $\sim 100$ colony-forming units $\mathrm{Mtb}$ by the aerosol route. On day 30 post-challenge, formalin-fixed lung samples were stained with hematoxylin and eosin (H\&E) or CD3 (red) B220 (green), and the area occupied by granuloma as (a) inflammatory lesions and (b) B-cell lymphoid follicles quantified using the morphometric tool of the Zeiss Axioplan microscope. Representative pictures of (c-top panel) inflammatory lesions and (c-bottom panel) B-cell lymphoid follicles are shown. Original magnification for H\&E sections, $\times 100$, B-cell follicles, $\times 200$. Ifng ${ }^{-1-}$ mice were vaccinated, rested, Mtb-infected, and treated with isotype control antibody or treated with IL-17-neutralizing antibody between day 5 and day 21 (100 $\mu \mathrm{g}$ per mouse every $48 \mathrm{~h}$ ), and killed on day 30 post-infection. Formalin-fixed samples were stained with H\&E or CD3 (red) B220 (green), and the area occupied by (d) inflammatory lesions/ lung lobe and (e) average size of B-cell lymphoid follicles harboring $C D 3^{+}$lymphocytes was quantified by using the morphometric tool of the Zeiss Axioplan microscope. Representative figures showing (f-top panel) inflammatory lesions and (f-bottom panel) B-cell lymphoid follicles are shown. The data points represent the mean ( \pm s.d.) of values from $4-6$ mice $(\mathbf{a}-\mathbf{f}) .{ }^{\star \star} P \leqslant 0.005,{ }^{\star \star \star} P \leqslant 0.0005$. One experiment representative of two is shown.

IL-17 in mucosally vaccinated mice was linked to efficient CXCL13 induction and the subsequent attraction of CXCR5bearing $\mathrm{T}$ cells within TB inflammatory lesions. In support of our hypothesis, we found that CXCL13 mRNA (Figure 4a, upper panel) and protein (Figure 4a, lower panel) was expressed and localized within iBALT found in lungs of vaccinated B6 and Ifng ${ }^{-/-}$mice but was poorly expressed within the loosely organized cellular aggregates formed in lungs of vaccinated $I l 17^{-I-}$ mice. Quantification of CXCL13 mRNA within inflammatory lesions further confirmed these findings (Figure 4b). Furthermore, the expression of CXCL13 mRNA (Figure 4c, upper panel; d) and protein (Figure 4c, lower panel) 

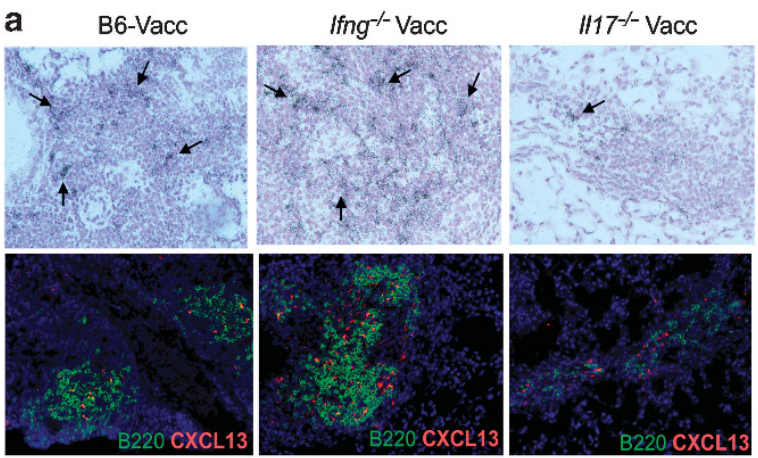

b

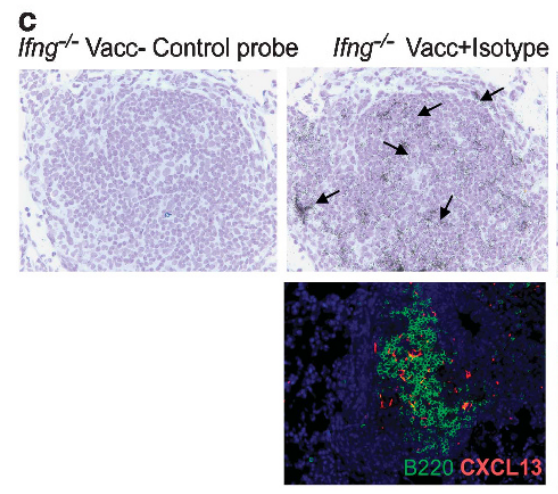

Ifng- Vacc+olL-17A

d
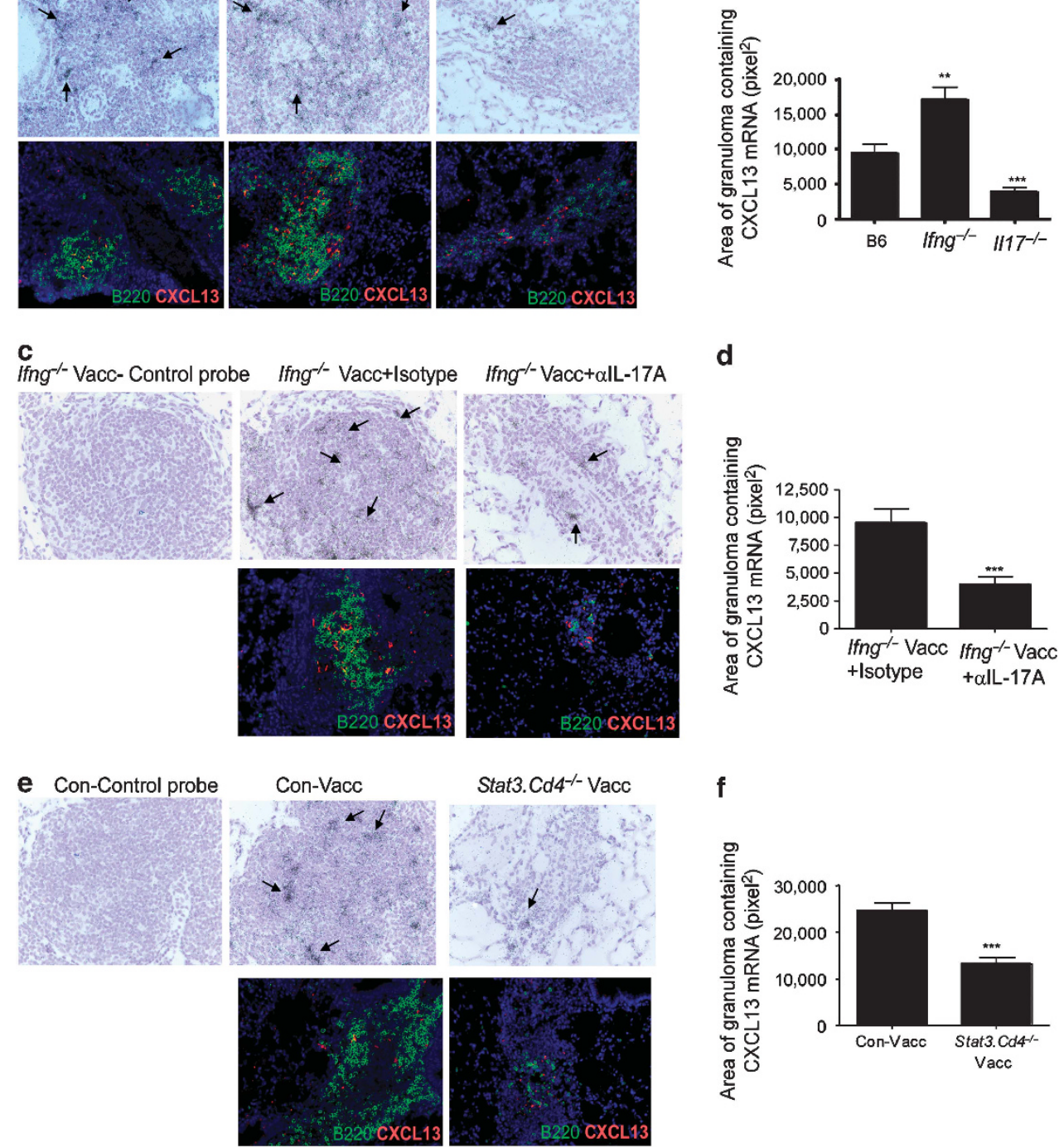

f

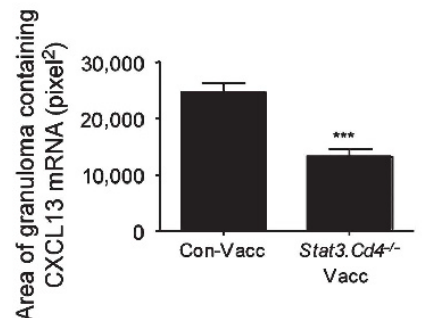

Figure 4 Interleukin (IL)-17 promotes C-X-C motif chemokine ligand 13 (CXCL13) expression within tuberculosis inflammatory lesions and mediates vaccine-induced protection against Mycobacterium tuberculosis (Mtb) infection. (a-d) B6, Ifng ${ }^{-/-}$and $1 / 17^{-/-}$were mucosally vaccinated with ESAT6 ${ }_{1-20}$ (early secreted antigenic target) in combination with LT-Ilb (type II heat labile enterotoxin), rested, challenged with $M$ tb, and killed on day 30 post-infection. Ifng ${ }^{-/}$mucosally vaccinated mice rested for 30 days were challenged with $M$ tb and treated with isotype antibody or IL-17 neutralizing antibody as described in Figure 2f. Littermate control mice (Con-Vacc), Stat3.Cd4 ${ }^{-/}$mice were mucosally vaccinated, rested, challenged with $M t b$, and killed on day 30 post-infection (e, f). Formalin-fixed, paraffin-embedded lung sections from the above groups were assayed for $C X C L 13$ mRNA localization by in situ hybridization using a murine CXCL13 mRNA probe or control probe (a, c, e-upper panels). Areas containing CXCL13 mRNA expression within inflammatory lesions was quantified as described under Materials and methods (b, d, f). Formalin-fixed, paraffin-embedded lung sections from the above groups were assayed using immunofluorescence for spatial detection of CXCL13 protein (a, c, e-lower panels). Original magnification for CXCL13 ISH sections, $\times 400$, immunofluorescent sections, $\times 200$. Pictures are representative of staining observed in the lungs of mice within the group $\left(n=4-6\right.$ mice) $(\mathbf{a}, \mathbf{c}, \mathbf{e})$. The data points represent the mean $\left( \pm \mathrm{s}\right.$.d.) of values from $4-6$ mice $(\mathbf{b}, \mathbf{d}, \mathbf{f})$. ${ }^{\star \star} P \leqslant 0.005$, ${ }^{* \star *} P \leqslant 0.0005$. One experiment representative of two is shown. IFN, interferon; STAT, signal transducer and activator of transcription factor.

in the inflammatory lesions of vaccinated $\mathrm{Ifng}^{-/-}$mice was lost upon neutralization of IL-17. In addition, reduced levels of CXCL13 mRNA (Figure 4e, upper panel), reduced area of granulomas containing CXCL13 mRNA (Figure 4f), and reduced expression of CXCL-13 protein (Figure 4e, lower panel) were detected within poorly organized inflammatory lesions of vaccinated Stat3.Cd4 ${ }^{-/}$mice. Vaccine-induced IL-17 is required for early CXCL9 induction and CXCR3- expressing Th1 recruitment into lungs of parenterally vaccinated $M t b$-challenged mice. ${ }^{10}$ However, we observed similar pulmonary CXCL9 mRNA expression in mucosally vaccinated B6 and $I l 17^{-/-} M t b$-challenged lungs (Supplementary Figure S3). As expected, pulmonary CXCL9 mRNA expression was lost in mucosally vaccinated Ifng $^{-/-} M t b$-challenged lungs (Supplementary Figure S3). Altogether, these data suggest that unlike parenteral 
immunization models, mucosal vaccine-induced IL-17 is critical for induction of CXCL13 but not CXCL9 during TB recall responses.

The absence of IL-17 did not impact accumulation of ESAT6 1-20 -specific Th1 cells in the lungs (Figure 2). In addition, we found similar accumulation of activated $\mathrm{CD}^{+} \mathrm{T}$ cells (Supplementary Figure S4a) and similar numbers of activated $\mathrm{CD}^{+}{ }^{+} \mathrm{T}$ cells expressing the chemokine receptors, CXCR5 and CXCR3 (Supplementary Figure S4b,c) in vaccinated $M t b$-challenged lungs of $\mathrm{B} 6$ and $I l 17^{-/-}$mice. Comparable numbers of activated CD $4^{+} \mathrm{T}$ cells producing proinflammatory cytokines such as IFN $\gamma$ (Supplementary Figure S4d), TNF $\alpha$ (Supplementary Figure S4e), IL-2 (Supplementary Figure S4f), as well as the ability of activated $\mathrm{CD} 4{ }^{+} \mathrm{T}$ cells to coproduce these cytokines (data not shown) was also similar between B6 and $I l 17^{-/-}$-vaccinated $M t b$-challenged lungs. As expected, IL-17producing activated $\mathrm{CD} 4^{+} \mathrm{T}$ cells (Supplementary Figure S4g) and IL-17/IFN $\gamma$ coproducers (Supplementary Figure
S4h) were absent in the lungs of vaccinated $M t b$-challenged $I l 17^{-/-}$mice. Importantly, despite similar accumulation of activated $\mathrm{CD} 4{ }^{+} \mathrm{T}$ cells producing proinflammatory cytokines to the lung, $\mathrm{T}$ cells failed to localize within lymphoid structures and resulted in increased T-cell perivascular cuffing (Figure 5a) and coincided with decreased T-cell localization within the granulomas (Figure 5b) in $I l 17^{-1-}$-vaccinated $M t b$-infected lungs. Based on these data, we hypothesized that vaccineinduced, activated cytokine-producing $\mathrm{CD}^{+} \mathrm{T}$ cells accumulate in the $M t b$-challenged lungs, but as they do not localize within lung inflammatory lesions, this may result in reduced activation of lung macrophages and therefore loss of vaccine-induced control. Consistent with this hypothesis, despite similar numbers of macrophages at early time points in B6 unvaccinated $\left(2.1 \times 10^{5} \pm 2.8 \times 10^{4}\right)$ and vaccinated $\left(2.6 \times 10^{5} \pm 2.4 \times 10^{4}, \quad P=0.2138\right.$ between unvaccinated and vaccinated B6 mice) $M t b$-challenged lungs, macrophages were less activated in unvaccinated $M t b$-challenged lungs when compared with macrophages in

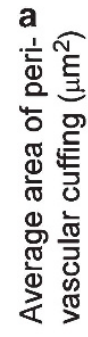

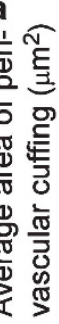
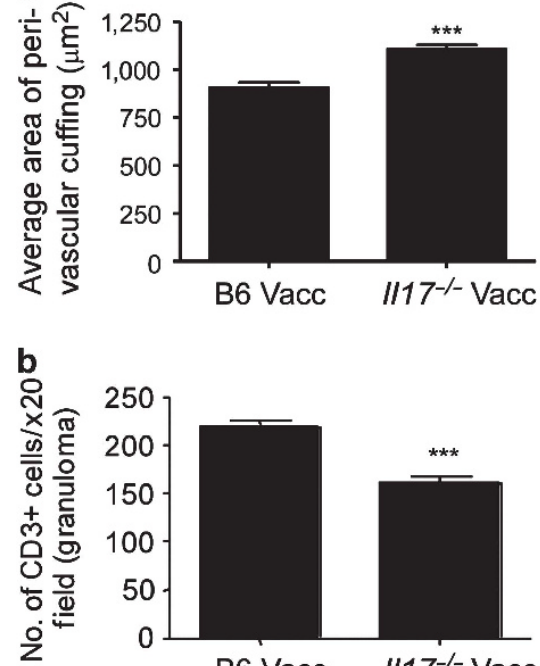

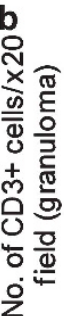

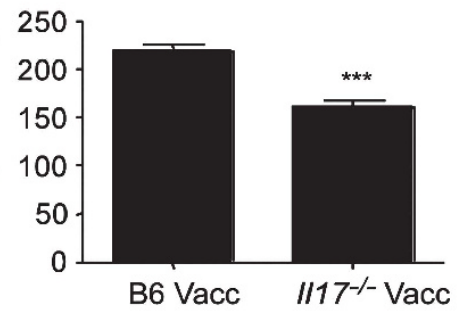

d

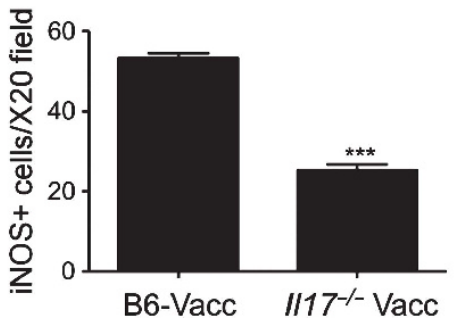

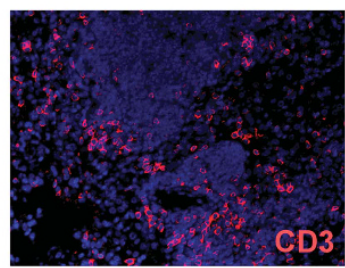

B6-Vacc

c

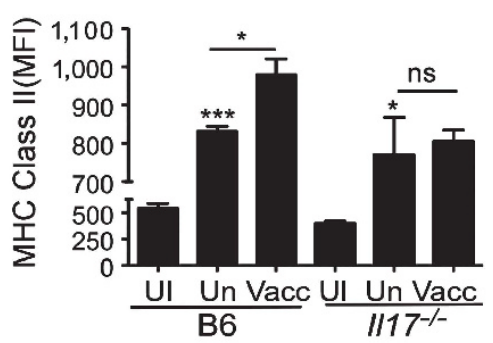

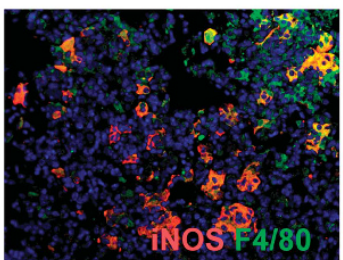

B6-Vacc

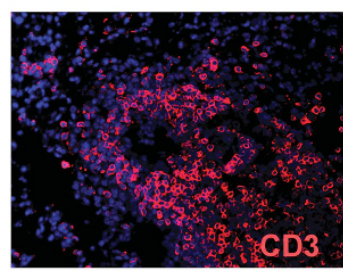

$1117^{-/-}$Vacc $1117^{-/}$

Figure 5 Absence of interleukin (IL)-17 results in impaired lung macrophage activation during vaccine-induced immunity. B6, and $/ 117^{-/-}$were mucosally vaccinated with ESAT6 ${ }_{1-20}$ (early secreted antigenic target) in combination with LT-IIb (type II heat labile enterotoxin), rested, and challenged with Mycobacterium tuberculosis (Mtb) as described in Figure 1. Formalin-fixed, paraffin embedded lung sections from day $30 \mathrm{Mtb}$-challenged lungs were analyzed by immunofluorescence for T-cell perivascular cuffing $\left(\mathrm{CD}^{+}\right)$and quantified using the morphometric tool of the Zeiss Axioplan microscope, and (a) a representative image of typical T-cell perivascular cuffing is shown. (b) The number of CD3 + T cells within the granuloma were quantitated as described under Materials and methods. (c) The mean fluorescent intensity (MFI) of MHC (major histocompatibility complex) Class II I-A expression on lung macrophages was determined by flow cytometry on day 15 from mucosally vaccinated Mtb-infected mice (Vacc), unvaccinated $M t b-$ infected mice (Un), or uninfected mice (UI). (d) The number of F4/80 macrophages producing inducible nitric oxide synthase (iNOS) were enumerated on day 30 post-infection in formalin-fixed lungs by immunofluorescence and a representative picture shown. The data points represent the mean ( \pm s.d.) of values from 4-6 mice (a-d). ${ }^{\star} P \leqslant 0.05,{ }^{\star \star \star} P \leqslant 0.0005$. One experiment representative of two is shown. NS, not significant. 
vaccinated $M t b$-challenged lungs (Figure 5c). Importantly, despite comparable numbers of lung macrophages in $\mathrm{Il17}^{-/-}$. vaccinated $M t b$-challenged lung $\left(2.8 \times 10^{5} \pm 3.6 \times 10^{4}\right.$, $P=0.6130$ between B6 and $I l 17^{-/-}$-vaccinated lungs), early macrophage activation was compromised in the $I l 17^{-/-}$-vaccinated $M t b$-challenged lungs (Figure 5c). Consequently, fewer inducible nitric oxide synthase (iNOS)expressing macrophages were found within the lungs in vaccinated $M t b$-challenged $I l 17^{-/-}$mice (Figure 5d). We observed similar results in Stat3.Cd4 ${ }^{-1-}$-vaccinated $M t b$-infected mice (data not shown). These data show that IL-17 induces CXCL13 expression and promotes correct T-cell localization for optimal macrophage activation, two key events required for early $M t b$ control in mucosally vaccinated mice.

To confirm that CXCL13-dependent recruitment of T cells within inflammatory lesions was critical for vaccine-induced immunity, we mucosally vaccinated $\mathrm{Cxcl13}{ }^{-/-}$mice and found that the absence of CXCL13 resulted in loss of vaccineinduced immunity against TB (Figure 6a). Importantly, lack of CXCL13 did not result in impairment in generation of Th17 cells in the lung following mucosal vaccination (Figure 6b) but influenced lung inflammatory lesion size and lymphocytic infiltration (Figure $\mathbf{6 c}, \mathbf{e}$, upper panel), resulting in smaller B-cell follicles (Figure 6d,e, lower panel) in mucosally vaccinated $\mathrm{Mtb}$-challenged $\mathrm{Cxcl1}^{-/-}$mice when compared with B6-vaccinated Mtb-challenged mice. Importantly, we found that $\mathrm{T}$ cells did not localize within lymphoid structures and accumulated as perivascular cuffs (Figure 6e,f) and coincidently exhibited decreased localization within the tubercle granuloma (Figure $\mathbf{6 g}$ ). This decreased localization of $\mathrm{T}$ cells within the granuloma also coincided with decreased activation of macrophages in inflammatory lesions of Cxcl13 ${ }^{-/-}$-vaccinated $M t b$-infected mice (Figure 6h). These data together suggest that vaccine-induced IL-17-mediated induction of CXCL13 is essential for correct T-cell localization within TB inflammatory lesions, early macrophage activation, and vaccine-induced $M t b$ control.

\section{IL-17 but not IFN $\gamma$ treatment can improve vaccine-induced protection against $M$ tb challenge}

Our data demonstrate that the protection afforded in mucosally vaccinated mice is mediated through the enhanced production of IL-17, local CXCL13 production, and improved lymphoid structure formation. This is a novel and important observation, as efforts to improve Th1 responses alone have not improved vaccine-induced protection following $M t b$ challenge. ${ }^{2}$ Moreover, most studies only measure antigen-specific IFN $\gamma$ responses following $M t b$ challenge, ${ }^{12,17}$ and are thus likely to miss the critically important IL-17-producing Th17 cells. Based on these data, we treated mucosally vaccinated mice with recombinant IL-17 or IFN $\gamma$, following $M t b$ challenge and determined whether this would further enhance immune protection. Accordingly, we found that early exogenous IL-17 treatment in mucosally vaccinated B6 mice following $M t b$ challenge, further decreased lung bacterial burden, when compared with bacterial burden in control mucosally vaccinated mice, and mucosally vaccinated mice that received recombinant IFN $\gamma$ (Figure 7a). The improved protection in mucosally vaccinated mice that received exogenous IL-17 also coincided with improved granuloma formation (Figure $\mathbf{7 b}, \mathbf{d}$, upper panels), enhanced B-cell follicle formation (Figure $\mathbf{7 c}, \mathbf{d}$, middle panels), and enhanced production of CXCL13 (Figure 7d, lower panels). This also coincided with decreased T-cell perivascular cuffing in B6 mucosally vaccinated mice that received early IL-17 therapy (Figure 7e). Similarly, adenoviral overexpression of IL-23 in mucosally vaccinated B6 mice also improved protective outcomes upon $M t b$ challenge, when compared with mucosally vaccinated $M t b$-infected mice that received control vector (Figure 7f). We then addressed if mucosal boosting in mice parenterally vaccinated with Mycobacterium bovis Bacille Calmette-Guerin (BCG) would improve vaccineinduced immunity upon $M t b$ challenge. Despite similar levels of vaccine-induced responses seen in mice parenterally vaccinated with $M$. bovis BCG and mucosally vaccinated with $M t b$ antigen and adjuvant, we found that mucosal boosting in M. bovis BCG-vaccinated mice further improved upon vaccine-induced immunity in $M t b$-challenged mice (Figure $7 \mathrm{~g}$ ). These data, therefore, demonstrate that experimentally manipulating IL-17 for improvement of vaccine efficacy against $M t b$ can be an effective strategy that can lead to improved vaccines against TB.

\section{DISCUSSION}

$M t b$ latently infects one-third of the world's population, of which $10 \%(\sim 200$ million people) will develop active TB during their lifetime. Therefore, it is critical that we design effective vaccine strategies to limit rising numbers of infected individuals. Immunity to $\mathrm{TB}$ has been associated with induction of effective Th1 responses; ${ }^{1}$ however, an IFN $\gamma$-independent mechanism of protection against $M t b$ infection exists, as $M t b$-specific Th cells lacking IFN $\gamma$ or TNF $\alpha$ still confers protection in $M t b$-infected mice. ${ }^{4,18}$ Instead, IFN $\gamma$ may limit IL-17 production, chemokine induction, neutrophil accumulation, and coincident lung inflammation during TB. ${ }^{18}$ Our new data show that the IFN $\gamma$-independent mechanism of protection functional in a mucosal vaccination model against $M t b$ infection is an IL-17-dependent mechanism, a surprising and novel finding, considering that IL-17 is dispensable for primary immunity to $\mathrm{TB}^{8}{ }^{8}$ These data suggest that protective immune molecules in primary and memory responses to mucosal infections are distinct and need to be clearly defined before exploring ways to experimentally target them in vaccine strategies. Furthermore, our data projects the new finding that the role for IL-17 in mediating vaccineinduced protective responses in parenteral vs. mucosal vaccine models are also likely different. For example, previously, we had described that following parenteral vaccination, IL-23 is required to prime a population of lung-resident Th17 cells that upon $M t b$ infection induce CXCR-3 ligating chemokines such as CXCL9, CXCL10, and CXCL11 responsible for the attraction of Th1 cells to the lung and subsequent activation of 

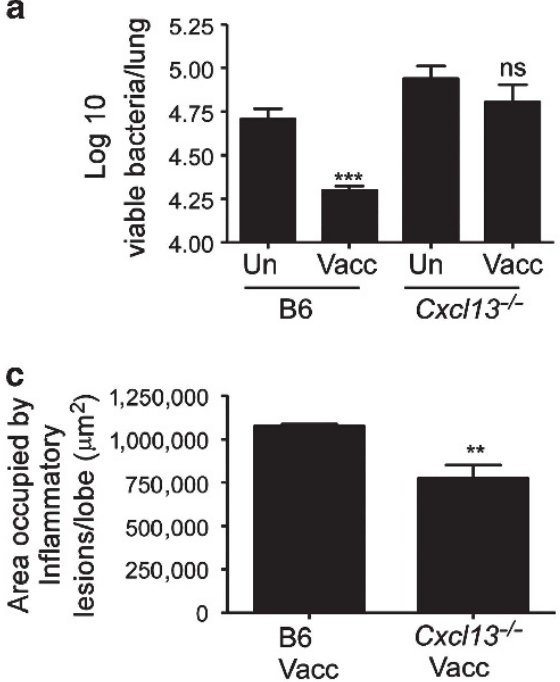

e

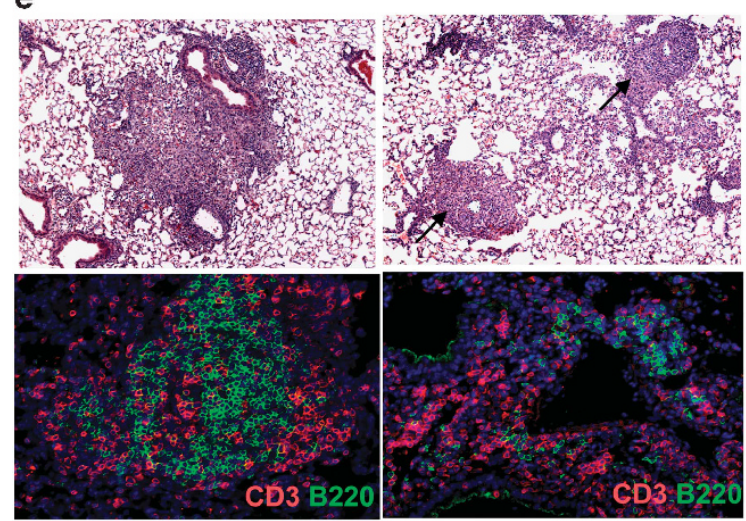

B6-Vacc

Cxcl13-- Vacc

b
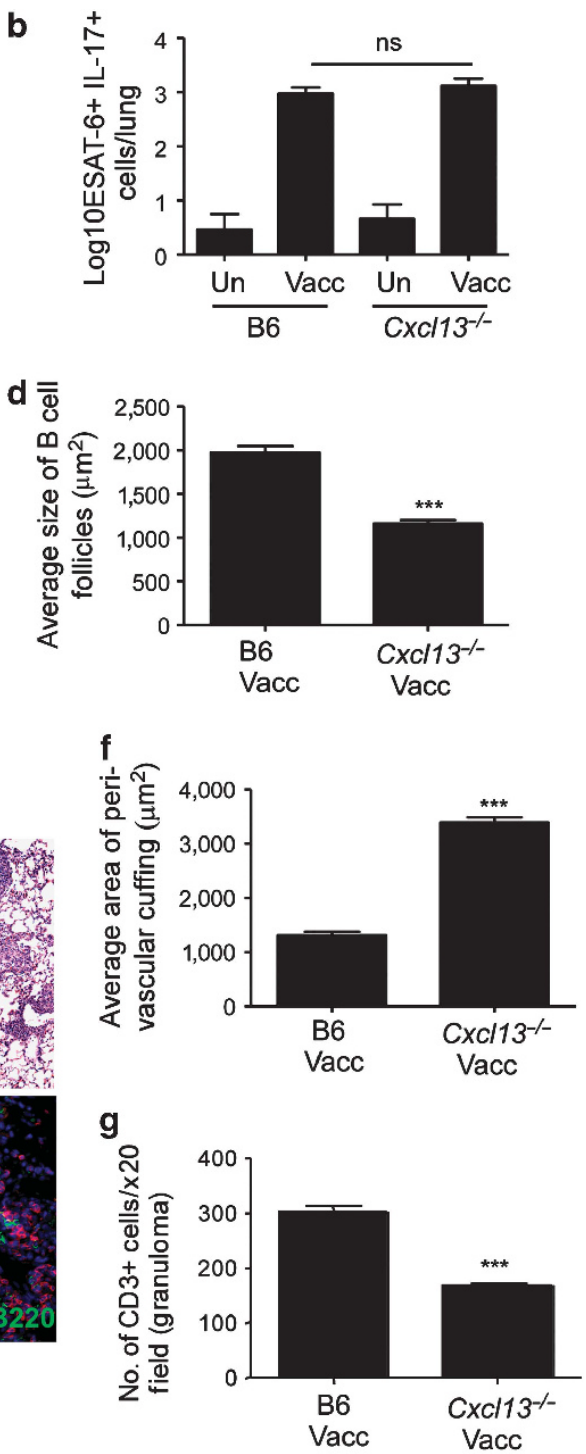

h

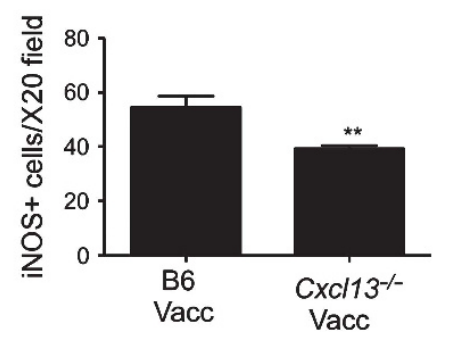

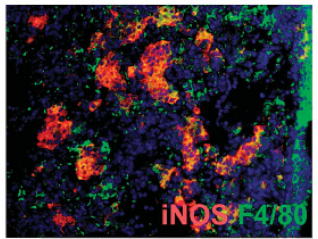

B6-Vacc

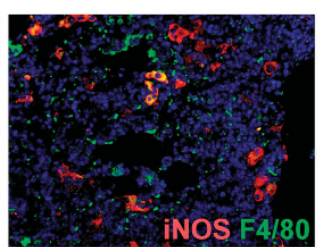

Cxcl13--Vacc

Figure 6 C-X-C motif chemokine ligand $13(\mathrm{CXCL} 13)$ is required for mucosal vaccine-induced immunity against tuberculosis. B6 and $\mathrm{CxC} / 13^{-/-}$mice were vaccinated with ESAT6 ${ }_{1-20}$ (early secreted antigenic target) in Lt-Ilb (type II heat labile enterotoxin), rested for 30 days, and challenged with Mycobacterium tuberculosis (Mtb) as described in Figure 1 and (a) the lung bacterial burden was determined. $(n=8-10$ mice combined over two

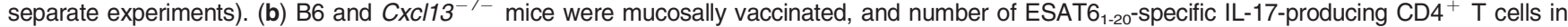
the lungs were determined by ELISpot on day 15 post-vaccination. Area occupied by (c) inflammatory lesions/lung lobe and (d) the average size of B-cell lymphoid follicles was quantified using the morphometric tool of the Zeiss Axioplan microscope in formalin-fixed day 30 Mtb-challenged lungs. Lung sections from $\mathrm{B} 6$ and $\mathrm{Cxc} / 13^{-{ }_{-}}$mucosally vaccinated $M$ tb-infected mice were stained with (e-top panel) hematoxylin and eosin or (e-bottom panel) CD3 (red) and B220 (green) on day 30 post-infection. Arrows point to perivascular cuffs in $\mathrm{Cxcl13^{-1 }}$ mice. (f) Formalin-fixed, paraffinembedded lung sections were analyzed by immunofluorescence for T-cell perivascular cuffing $\left(\mathrm{CD}^{+}{ }^{+}\right.$staining), $(\mathbf{g})$ the number of $\mathrm{CD} 3+\mathrm{T}$ cells that localized within the granuloma and $(\mathbf{h})$ the number of iNOS ${ }^{+}$(inducible nitric oxide synthase-positive) cells per inflammatory lesions. (f) T-cell perivascular cuffing was quantified in formalin-fixed lung sections $\left(\mathrm{CD}^{+}\right)$using the morphometric tool of the Zeiss Axioplan microscope. CD3+ $\mathrm{T}$ cells and F4/80-expressing macrophages producing iNOS were enumerated by immunofluorescence and representative pictures are shown $(\mathbf{g}, \mathbf{h})$. The data points represent the mean ( \pm s.d.) of values from $4-6$ mice $(\mathbf{b}-\mathbf{h}) .{ }^{* *} P \leqslant 0.005,{ }^{* \star *} P \leqslant 0.0005$. One experiment representative of two is shown. NS, not significant. 


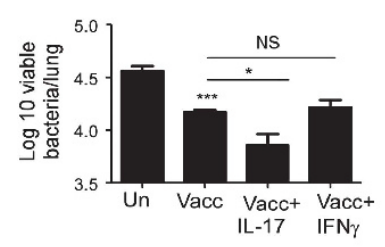

b

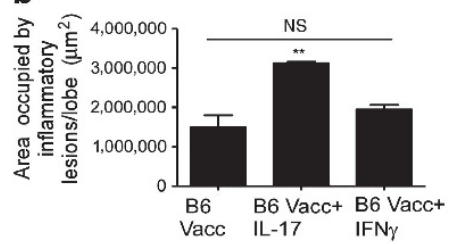

\section{C}

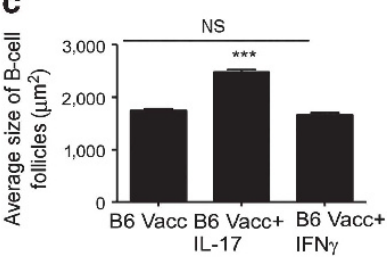

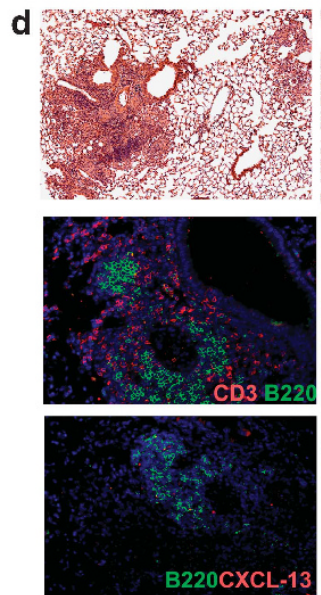

B6 Vacc
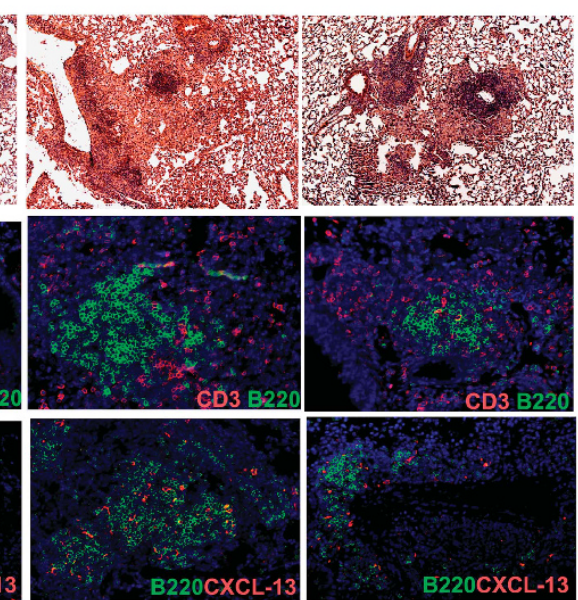

B6 Vacc+IL-17

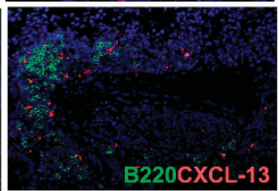

B6 Vacc+IFN $\gamma$

e

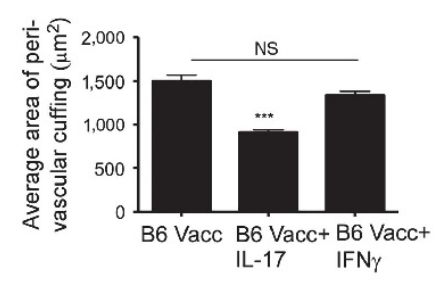

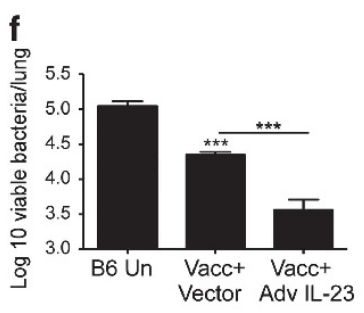

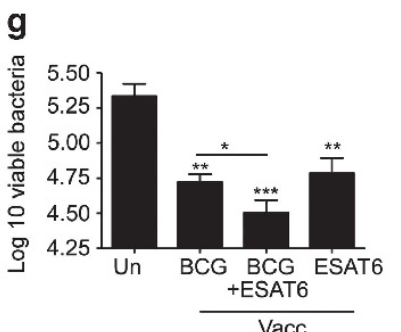

Figure 7 Interleukin (IL)-17 improves the protective efficacy of mucosal vaccination following Mycobacterium tuberculosis (Mtb) challenge. B6 mice were mucosally vaccinated with ESAT6 ${ }_{1-20}$ (early secreted antigenic target) in LT-Ilb (type II heat labile enterotoxin), rested, and challenged with Mtb as described in Figure 1. Vaccinated $M$ tb-challenged mice either received phosphate-buffered saline, rlL-17 or recombinant interferon- $\gamma(\mathrm{rlFN} \gamma ; 1.5 \mu \mathrm{g}$ per mouse) intratracheally from day 5 to day 17 post-infection during recall response, and (a) the lung bacterial burden was determined on day 30 postvaccination. On day 30 post-infection, formalin-fixed, paraffin-embedded lung sections were stained with hematoxylin and eosin (H\&E) or CD3 (red), and B220 (green). Area occupied by (b) inflammatory lesions/lung lobe and (c) B-cell lymphoid follicles was quantified using the morphometric tool of the Zeiss Axioplan microscope. The representative figure showing (d-top panel) a typical inflammatory lesions, (d-middle panel) B-cell follicle and (d-bottom panel) C-X-C motif chemokine ligand 13 (CXCL13) expression within B-cell follicles is included. (e) The average area of perivascular cuffing from the above-mentioned groups were quantified. One group of mucosally vaccinated B6 mice received control adenovirus expressing luciferase vector (Vacc + Vector), while a second group received adenovirus overexpressing IL-23 (Vacc + Ad IL-23) $\left(5 \times 10^{8}\right.$ plaque-forming units) on the day of vaccination. Mucosally vaccinated and unvaccinated mice were rested and challenged with $M t b$ and (f) the lung bacterial burden was determined on day 30 post-infection. B6 mice were either subcutaneously vaccinated with $1 \times 10^{6} \mathrm{M}$. bovis Bacille Calmette-Guerin (BCG), mucosally vaccinated, or subcutaneously vaccinated with M. bovis BCG followed by a period of rest for 30 days and boosted mucosally as described under Methods. All groups of mice were then rested for 30 days and challenged with $M t b$ as described in Figure 1 and (g) the lung bacterial burden was determined on day 30 postinfection. Original magnification for H\&E sections, $\times 100$; immunofluorescent sections, $\times 200$. The data points represent the mean ( \pm s.d.) of values from 4-6 mice (a-g). ${ }^{*} P \leqslant 0.05,{ }^{* \star} P \leqslant 0.005,{ }^{* * \star} P \leqslant 0.0005$. One experiment representative of two is shown. NS, not significant.

macrophages for $M t b$ control. ${ }^{10}$ When compared with this parenteral model of vaccination, our new data show that potent mucosal adjuvants and mucosal routes of vaccination can also elicit enhanced generation of lung-resident Th17 cells; however, in this model the protection is IFN $\gamma$-independent but instead is dependent on IL-17-induced CXCL13 expression and strategic localization of $\mathrm{T}$ cells within inflammatory lesions to activate macrophages to control $M t b$. This is an important and novel finding, as the absence of IL-17 completely abrogated vaccine-induced protection in the mucosal model. We recently showed that IL-17 induces expression of chemokines CCL19 and CXCL13 in the neonate lung and mediates generation of iBALT structures following lipopolysaccharide-induced inflammation. ${ }^{7}$ Furthermore, lung fibroblasts can directly respond to IL-17 and induce CXCL13 expression in vitro. ${ }^{8}$ Consistent with these previous observations, our new findings presented here show that vaccine-induced Th17 cells produce IL-17 that drives CXCL13 induction within the lung parenchyma, promoting the correct T-cell localization within the inflammatory lesions, in order to maximally activate 
macrophages and optimize immune protection. It is interesting that the absence of IL-17 does not impact primary immunity to $\mathrm{TB},{ }^{8}$ while the absence of CXCL13 increases susceptibility to primary $M t b$ infection. ${ }^{6}$ These observations suggest the existence of IL-17-independent mechanisms of CXCL13 induction during primary $M t b$ infection. In mucosally vaccinated Ifng ${ }^{-1-}$ mice, despite loss of CXCL9 expression, IL-17-dependent production of CXCL13 is intact and helps to localize T cells within TB inflammatory lesions for adequate vaccine-induced control. We have detected $\mathrm{CXCR}^{+}{ }^{+} \mathrm{CD} 4^{+} \mathrm{T}$ cells that express IFN $\gamma$, TNF- $\alpha$, and IL-2, which potentially could accumulate inside inflammatory lesions (data not shown). In this location, CXCR5-expressing $\mathrm{T}$ cells can produce a variety of Th1 effector cytokines to induce iNOS-dependent and iNOS-independent mechanisms, critical for macrophage activation and vaccine-induced protection. We observed that depletion of IL-17 in Ifng ${ }^{-/-}$-vaccinated mice notably reduced CXCL13 expression, impaired T-cell localization within inflammatory lesions, and severely compromised vaccine-induced protection. These collective data confirms that production of IL-17, but not IFN $\gamma$, is the critical first step in the downstream events that mediate vaccine-induced immunity against TB.

Recently, several adjuvants, including oil-in-water nanoemulsions, ${ }^{19}$ cholera toxin, ${ }^{20,21}$ polyelectrolyte microcapsules, ${ }^{22}$ have been shown to induce potent Th17 responses when delivered mucosally. Similarly, our data demonstrate that delivery of $M t b$-specific antigens in the presence of LT-IIb as adjuvant enhances lung-resident Th17 responses. Recently, it has become clear that following exposure to adjuvants, both $\alpha \beta$ $\mathrm{T}$ cells as well as innate cells such as $\gamma \delta$ T cells can produce IL$17 .{ }^{23}$ However, we found that following mucosal vaccination of ESAT6 $6_{1-20}$ with LT-IIb, $\alpha \beta$ T cells were the primary cellular source of IL-17 in the lung (data not shown). These data suggest that mucosal vaccination with LT-IIb may induce production of polarizing cytokines such as IL-6, TGF $\beta$, and IL-23 in mucosal DCs to induce Th17 responses. ${ }^{20-22}$ Accordingly, DCs isolated from lungs of mice mucosally administered ESAT6 ${ }_{1-20}$ in LT-IIb induce mRNA for IL-12p40 (data not shown). These data suggest that the mucosal route of vaccination in combination with an appropriate mucosal adjuvant can be an effective strategy to induce lung-resident $M t b$-specific vaccine-induced Th17 responses and improve upon vaccine-induced immunity against TB. However, excess IL-17 induced in response to repeated mycobacterial vaccinations ${ }^{24}$ or in the absence of IFN $\gamma$ signaling ${ }^{18}$ can result in severe lung pathology. Therefore, the pathological vs. protective role of IL-17 should be carefully explored before targeting IL-17 in vaccine strategies for TB.

Recent studies show that pulmonary infection with $M t b$ triggers the formation of lymphoid aggregates associated with TB granuloma. ${ }^{25}$ Our data in the current paper expand these findings and show that mucosal vaccine-induced Th17 cells induce enhanced formation and organization of lymphoid structures within TB inflammatory lesions, suggesting that protection in mucosally vaccinated mice coincides with the increased cellular complexity of iBALT structures. Consistent with these data, the absence of vaccine-induced immunity in $I l 17^{-/-}$mice correlates with the reduced expression of CXCL13, poor generation of B-cell follicles, poorly formed lymphocytic infiltrates, and increased T-cell perivascular cuffing. These data together suggest that IL-17-dependent iBALT formation and organization are required to facilitate the cross-talk between $\mathrm{T}$ cells and macrophages inside granulomas and to enhance $M t b$ control. Together, our recent studies in primary $M t b$ infection $^{6,8}$ and in mucosal vaccine-induced immunity described here support the idea that iBALT is critical for protection against $\mathrm{TB}$. In both the models, CXCL13 expression and accumulation of CXCR5 ${ }^{+} \mathrm{T}$ cells within the granuloma is fundamental for quick and optimal macrophage activation. Interestingly, in primary $M t b$ infection, IL-17 is not required for protection, ${ }^{8}$ suggesting that other factors can induce CXCL13 and mediate protection. Based on our data, it is tempting to speculate that mucosal boosting may enhance Th17 lung-resident cells in pre-immunized individuals, improve iBALT formation, and provide protection against TB, thereby decreasing global TB burdens. Taken together, these results have far-reaching implications for the design of future vaccines for TB.

\section{METHODS}

Mice. C57BL/6 (B6), Cxcl13 $3^{-/-}$and Ifng ${ }^{-/-}$mice were purchased from The Jackson Laboratory (Bar Harbor, ME), $I l 17^{-/-}$mice were obtained on the B6 background, ${ }^{26}$ Stat 3.C $d 4^{-1-}$ mice were obtained from Indiana University School of Medicine. ${ }^{27}$ Experimental mice were age-and sex-matched and used between the ages of 6-8 weeks. All mice were maintained and used in accordance with the approved University of Pittsburgh IACUC guidelines.

Cloning and purification of holotoxins (LT-Ilb). Engineering of plasmid encoding wild-type LT-IIb has been described. ${ }^{14}$ Plasmidencoded, his-tagged HLTs were purified from the periplasmic extracts of cultures of Escherichia coli DH5 $\alpha$ F'kan (Life Technologies, Gaithersburg, MD) as described before. ${ }^{14}$ Precipitated proteins were dialyzed to remove salts and low molecular weight molecules. His-tagged HLTs in the dialysate were initially resolved by nickel affinity chromatography using His Bind resin (Novagen, Madison, WI). Peak fractions from the nickel affinity chromatography demonstrated by SDS-PAGE (sodium dodecyl sulfate-polyacrylamide gel electrophoresis) of the eluted fractions were pooled and subsequently applied to a Sephacryl S-100 gel filtration column (Pharmacia, Piscataway, NJ) connected to an AKTAfplc (Pharmacia). HLTs obtained from gel filtration were resolved by SDS-PAGE to confirm homogeneity and by immunoblotting using anti-LT-IIb antibodies to confirm identity. Finally, purified proteins were analyzed for potentially contaminating endotoxin (lipopolysaccharide) using a quantitative Limulus amoebocyte lysate assay (Charles River Endosafe, Charleston, SC). HLTs were essentially free of lipopolysaccharide

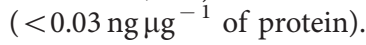

Vaccinations and experimental infections. ESAT6 $6_{1-20}$ peptide (133 mg) was mixed with LT-IIb HLT ( $1 \mu \mathrm{g})$, and unanesthetized mice were mucosally vaccinated intranasally three times at 2 -week intervals. In some experiments, adenovirus overexpressing IL-23 or control adenovirus expressing luciferase vector ${ }^{28}$ was delivered once $\left(5 \times 10^{8}\right.$ plaque-forming units) intratracheally on the day of vaccination. M. bovis BCG Pasteur was grown in Proskauer Beck medium containing $0.05 \%$ Tween 80 to mid-log phase and then frozen in 1-ml aliquots at $-80^{\circ} \mathrm{C}$. Bacterial stocks were plated on $7 \mathrm{H} 11$ agar plates to 
calculate colony-forming units (CFU). Mice were vaccinated subcutaneously with $1 \times 10^{6} \mathrm{CFU}$ of $M$. bovis BCG in phosphate-buffered saline and after 30 days some mice were mucosally vaccinated with two doses of Ag85B 240-254 $_{\text {peptide }}(133 \mathrm{mg})$ mixed with LT-IIb HLT $(1 \mu \mathrm{g})$. All vaccinated mice were rested for 30 days following which they were challenged with $M t b \mathrm{H} 37 \mathrm{Rv}$. The H37Rv strain of $M t b$ was grown in Proskauer Beck medium containing 0.05\% Tween-80 to mid-log phase and frozen in $1 \mathrm{ml}$ aliquots at $-70^{\circ} \mathrm{C}$. For $\mathrm{Mtb}$ aerosol infections, animals were infected with $100 \mathrm{CFU}$ of bacteria using a Glas-Col airborne infection system as described before. ${ }^{10}$ In some experiments, mice were treated intraperitoneally with either $100 \mu \mathrm{g}$ of $\alpha \mathrm{IL}-17 \mathrm{~A}$ (clone 50,104) or isotype control (clone 54,447; R\&D Systems, Minneapolis, MN) from day 9 through day 21 post-infection. In other experiments, mice were treated with recombinant IL-17A (rIL-17A) or IFN $\gamma(\mathrm{rIFN} \gamma)(\mathrm{R} \& \mathrm{D}$ Systems) intratracheally at a dose of $1.5 \mu \mathrm{g}$ in $50 \mu \mathrm{l}$ saline per mouse ${ }^{10}$ from day 5 to day 17 post-infection every $48 \mathrm{~h}$. Bacterial burden was estimated by plating the lung homogenates on 7H11 agar plates.

Lung cell preparation. Lung cell suspensions were prepared as described and single cells were used for ELISpot or flow cytometric analyses. $^{9-10}$

Flow cytometry. Single-cell suspensions were stained with fluorochrome-labeled antibodies specific for CD3 (145-2C11), CD4 (RM4-5), CXCR5 (2G8), IFN $\gamma$ (XMG1.2), IL-17 (TC11-18H10), TNF $\alpha$ (MP6-XT22), IL-2 (JES6-5H4), CD44 (IM7), CD11c (HL3), CD11b (M1/70), CXCR3 (173), MHC Class II (M5/114.15.2), or isotype control antibodies. For intracellular analyses, cells were stimulated with phorbol myristate acetate $\left(50 \mathrm{ng} \mathrm{ml}^{-1}\right)$ and ionomycin (750 ng ml ${ }^{-1}$; Sigma-Aldrich, St Louis, MO) in the presence of Golgistop (BD Pharmingen, San Diego, CA). Cells were then surface stained, permeabilized with Cytofix-Cytoperm solution (BD Pharmingen), and stained for relevant cytokines. Cells were collected using a Becton Dickinson FACS Aria flow cytometer using FACS Diva software (BD, Franklin Lakes, NJ). Cells were gated based on their forward by side-scatter characteristics and the frequency of specific cell types was calculated using FlowJo (Tree Star, Ashland, OR). The mean fluorescent intensity was also calculated to determine the expression levels of molecules using FlowJo (Tree Star).

Detection of cytokine-producing cells by ELISpot assay. Detection of antigen-specific IFN $\gamma$ - and IL-17-producing cells was carried out by using an ELISpot assay. ${ }^{10}$ In brief, cell culture plates were coated overnight with monoclonal purified anti-mouse IFN $\gamma$ (Clone R4-6A2; eBiosciences, San Diego, CA) or monoclonal purified anti-mouse IL-17 (clone 50,101; R\&D Systems) in phosphate-buffered saline. Cells were seeded at an initial concentration of $2-5 \times 10^{6}$ cells per well and doubling dilutions made. Irradiated B6 splenocytes were used as antigen-presenting cells. ESAT- $6_{1-20}$ peptide was used as antigen for vaccinated mice and mouse rIL-2 (Sigma-Aldrich; $10 \mathrm{U} \mathrm{ml}^{-1}$ ) was added to all wells. Plates were washed and biotinylated anti-mouse IFN $\gamma$ (clone XMG 1.2; eBiosciences) or biotynylated anti-mouse IL-17 antibody (clone eBio17B7) was used to detect the captured cytokine. Spots were enumerated by using the CTL-Immuno Spot analyzer (Cellular Technology Limited, Shaker Heights, $\mathrm{OH}$ ). The frequency of responding cells were determined and applied to the number of cells per sample to generate the total number of responding cells per organ.

Immunohistochemistry. Lung lobes were instilled with $10 \%$ neutralbuffered formalin and embedded in paraffin. Lung sections were stained with hematoxylin and eosin stain, and inflammatory features were evaluated by light microscopy (Research Histology Core, University of Pittsburgh). For immunofluorescent staining, formalinfixed, lung sections were cut, immersed in xylene to remove paraffin, and then hydrated in alcohol, 96\% alcohol, and phosphate-buffered saline. Antigens were unmasked with a DakoCytomation Target Retrieval Solution and non-specific binding was blocked with $5 \%(\mathrm{v} / \mathrm{v})$ normal donkey serum and Fc block (BD Pharmingen). Endogenous biotin (Sigma-Aldrich) was neutralized by adding first avidin, followed by incubation with biotin. Sections were probed with, anti-B220 to detect B cells (Clone RA3-6B2, BD Pharmingen), anti-CXCL13 (Clone 143614, R \& D Biosystems) and anti-CD3 to detect T cells (Clone M-20, Santa Cruz Biotechnology, Santa Cruz, CA) in the inflammatory lesions. iNOS-producing macrophages were identified using goat antiNOS2 (M-19-G, Santa Cruz Biotechnology) and rat F4/80 (MCA497GA, Serotec). Inflammatory lesions and B-cell follicles were outlined with the automated tool of the Zeiss Axioplan 2 microscope (Carl Zeiss, Thornwood, NY) and average size in squared microns were calculated. iNOS + cells in three random $\times 20$ fields were enumerated per lung ( $n=5$ lungs), and the average was calculated. In all, 3-5 granulomas per lobe section in each group were randomly chosen to quantify local CXCL13 mRNA expression or granuloma T-cell infiltration. in situ hybridization signal was quantified inside granulomas with Image J (NIH, Bethesda, MD), using the same threshold for all the analysis (100) and differences in the average area occupied by CXCL13 mRNA were graphed in GraphPad Prism 5 (La Jolla, CA). Infiltrating $\mathrm{T}$ cells in granulomatous structures were counted with the automated tool of the Axioplan Zeiss microscope in 3-5 randomly picked $\times 200$ fields. Samples were analyzed in a blinded fashion.

In situ hybridization. Mouse CXCL13 cDNA was reverse transcriptase-PCR amplified with primers BFJ. mCXCL13_F1 (59-GAACTCCACCTCCAGGCAGA-39) and BFJ. mCXCL13_R1 (59-CTTTTGA GATGATAGTGGCT-39). PCR products were ligated to the pGEM-T vector (Promega, Madison, WI) and DNA sequenced. The GEMT-CXCL13 plasmid was linearized by restriction digest. Gene-specific riboprobes were synthesized by in vitro transcription using a Maxiscript SP6/T7 kit (Ambion), and unincorporated nucleotides were removed using RNA Mini Quick Spin Columns (Roche, Indianapolis, IN). Paraffin-embedded tissue specimens were immersed with xylene for deparaffinization and rinsing in ethanol. In situ hybridization with 35 S-labeled riboprobes was performed at $50{ }^{\circ} \mathrm{C}$ overnight with $0.1 \mathrm{M}$ dithiothreitol included in the hybridization mix. CXCL9 mRNA was detected as previously described. ${ }^{29}$ Tissue sections were coated with NTB-2 emulsion (Kodak, Rochester, NY) and exposed at $10{ }^{\circ} \mathrm{C}$ for 10 days. The sections were counterstained with hematoxylin (Vector Laboratories, Burlingame, CA) and mounted with Permount (Fisher Scientific, Pittsburgh, PA). Images were visualized using an Olympus BX41 microscope (Olympus, Philadelphia, PA) and captured using a SPOT RT3 digital camera (Diagnostics Instruments, Sterling Heights, MI).

Statistical analysis. Differences between the means of groups were analyzed using the two-tailed Student's $t$-test in GraphPad Prism 5.

SUPPLEMENTARY MATERIAL is linked to the online version of the paper at http://www.nature.com/mi

\section{ACKNOWLEDGEMENTS}

This work was supported by Children's Hospital of Pittsburgh, NIH grants Al083541 and HL105427 to S.A.K., HL69409 to T.D.R., DE13833 to T.D.C., and Al060422 to T.A.R., Department of Medicine, University of Rochester and Al91036 to J.R.-M, a Research Advisory Committee Grants from Children's Hospital of Pittsburgh of the UPMC Health System to S.R.S and Y.L. We thank Dr Iwakura, University of Japan, for $I 117^{-1-}$ for breeders and Dr Kaplan, Indiana University School of Medicine for Stat3.Cd4 ${ }^{-1}$ breeders and Hillary Cleveland for mice breeding. We also thank Dr John Alcorn for critical reading of the manuscript.

\section{DISCLOSURE}

The authors declared no conflict of interest.

c 2013 Society for Mucosal Immunology 


\section{REFERENCES}

1. Cooper, A.M. \& Khader, S.A. The role of cytokines in the initiation, expansion, and control of cellular immunity to tuberculosis. Immunol. Rev. 226, 191-204 (2008).

2. Leal, I., Smedegard, B., Andersen, P. \& Appelberg, R. Failure to induce enhanced protection against tuberculosis by increasing T-cell-dependent interferon-gamma generation. Immunology 104, 157-161 (2001).

3. Cowley, S.C. \& Elkins, K.L. CD4 + T cells mediate IFN-gammaindependent control of Mycobacterium tuberculosis infection both in vitro and in vivo. J. Immunol. 171, 4689-4699 (2003).

4. Gallegos, A.M. et al. A gamma interferon independent mechanism of CD4 T cell mediated control of $M$. tuberculosis infection in vivo. PLoS Pathog. 7 , e1002052 (2011).

5. Rangel-Moreno, J., Moyron-Quiroz, J.E., Hartson, L., Kusser, K. \& Randall, T.D. Pulmonary expression of CXC chemokine ligand 13, CC chemokine ligand 19, and CC chemokine ligand 21 is essential for local immunity to influenza. Proc. Natl. Acad. Sci. USA 104, 10577-10582 (2007).

6. Khader, S.A. et al. In a murine tuberculosis model, the absence of homeostatic chemokines delays granuloma formation and protective immunity. J. Immunol. 183, 8004-8014 (2009).

7. Rangel-Moreno, J. et al. The development of inducible bronchusassociated lymphoid tissue depends on IL-17. Nat. Immunol. 12 , 639-646 (2011).

8. Khader, S.A. et al. IL-23 is required for long-term control of Mycobacterium tuberculosis and B cell follicle formation in the infected lung. J. Immunol. 187, 5402-5407 (2011).

9. Khader, S.A. et al. IL-23 compensates for the absence of IL-12p70 and is essential for the IL-17 response during tuberculosis but is dispensable for protection and antigen-specific IFN-gamma responses if $\mathrm{IL}-12 \mathrm{p} 70$ is available. J. Immunol. 175, 788-795 (2005).

10. Khader, S.A. et al. IL-23 and IL-17 in the establishment of protective pulmonary CD4 $+\mathrm{T}$ cell responses after vaccination and during Mycobacterium tuberculosis challenge. Nat. Immunol. 8, 369-377 (2007).

11. Goonetilleke, N. et al. Enhanced immunogenicity and protective efficacy against Mycobacterium tuberculosis of Bacille Calmette-Guerin vaccine using mucosal administration and boosting with a recombinant modified Vaccinia virus Ankara. J. Immunol. 171, 1602-1609 (2003).

12. Chen, L., Wang, J., Zganiacz, A. \& Xing, Z. Single intranasal mucosal Mycobacterium bovis BCG vaccination confers improved protection compared to subcutaneous vaccination against pulmonary tuberculosis. Infect. Immun. 72, 238-246 (2004).

13. Wang, J. et al. Single mucosal, but not parenteral, immunization with recombinant adenoviral-based vaccine provides potent protection from pulmonary tuberculosis. J. Immunol. 173, 6357-6365 (2004).
14. Nawar, H.F., Arce, S., Russell, M.W. \& Connell, T.D. Mucosal adjuvant properties of mutant LT-Ila and LT-Illb enterotoxins that exhibit altered ganglioside-binding activities. Infect. Immun. 73, 1330-1342 (2005).

15. Cooper, A.M. et al. Disseminated tuberculosis in interferon gamma genedisrupted mice. J. Exp. Med. 178, 2243-2247 (1993).

16. Flynn, J.L. et al. An essential role for interferon gamma in resistance to Mycobacterium tuberculosis infection. J. Exp. Med. 178, 2249-2254 (1993).

17. Andersen, C.S. et al. The combined CTA1-DD/ISCOMs vector is an effective intranasal adjuvant for boosting prior Mycobacterium bovis BCG immunity to Mycobacterium tuberculosis. Infect. Immun. 75, 408-416 (2007).

18. Nandi, B. \& Behar, S.M. Regulation of neutrophils by interferon-\{gamma\} limits lung inflammation during tuberculosis infection. J. Exp. Med. 208, 2251-2262 (2011).

19. Bielinska, A.U. et al. Induction of Th17 cellular immunity with a novel nanoemulsion adjuvant. Crit. Rev. Immunol. 30, 189-199 (2010).

20. Lee, J.B., Jang, J.E., Song, M.K. \& Chang, J. Intranasal delivery of cholera toxin induces th17-dominated T-cell response to bystander antigens. PLoS One 4, e5190 (2009).

21. Datta, S.K. et al. Mucosal adjuvant activity of cholera toxin requires Th17 cells and protects against inhalation anthrax. Proc Natl. Acad. Sci. USA 107, 10638-10643 (2010).

22. De Koker, S. et al. Biodegradable polyelectrolyte microcapsules: antigen delivery tools with Th17 skewing activity after pulmonary delivery. J. Immunol. 184, 203-211 (2010).

23. Reynolds, J.M., Angkasekwinai, P. \& Dong, C. IL-17 family member cytokines: regulation and function in innate immunity. Cytokine Growth Factor Rev. 21, 413-423 (2010).

24. Cruz, A. et al. Pathological role of interleukin 17 in mice subjected to repeated BCG vaccination after infection with Mycobacterium tuberculosis. J. Exp. Med. 207, 1609-1616 (2010).

25. Randall, T.D. Bronchus-associated lymphoid tissue (BALT) structure and function. Adv. Immunol. 107, 187-241 (2010).

26. Nakae, S. et al. Antigen-specific $\mathrm{T}$ cell sensitization is impaired in IL-17-deficient mice, causing suppression of allergic cellular and humoral responses. Immunity 17, 375-387 (2002).

27. Harris, T.J. et al. Cutting edge: An in vivo requirement for STAT3 signaling in TH17 development and TH17-dependent autoimmunity. J. Immunol. 179, 4313-4317 (2007).

28. Happel, K.I. et al. Pulmonary interleukin-23 gene delivery increases local T-cell immunity and controls growth of Mycobacterium tuberculosis in the lungs. Infect. Immun. 73, 5782-5788 (2005).

29. Aujla, S.J. et al. IL-22 mediates mucosal host defense against Gramnegative bacterial pneumonia. Nat. Med. 14, 275-281 (2008). 